Infekktionsgefahr für die Conjunctiva aber besonders groß ist. (Enges Zusammenleben mit gonorrhöekranken Erwachsenen.) Es werden allerdings durch diese beiden Momente nur ein großer Teil, aber nicht alle Fälle von Conjunctivitis gonorrhoica ohne Genitalgonorrhöe erklärt.

Zusammenfassung: $\mathrm{r}$. Die Häufigkeit der Conjunctivitis gonorrhoica infantum et adultorum (die Conjunctivitis gonorrhoica neonatorum wurde in dieser Arbeit nicht berücksichtigt) liegt in der Größenordnung von $2-4$ Fällen im Jahr auf I Million Einwohner.

2. Mehr als die Hälfte der Fälle betreffen Kinder unter I5 Jahren.

3. Während etwa drei Viertel der kindlichen Fälle Mädchen und ein Viertel Knaben betreffen, besteht bei Erwachsenen kein wesentlicher Unterschied zwischen Männern und Frauen. Das Überwiegen der Mädchen ist auf die Vulvovaginitis gonorrhoica zurückzuführen.

4. Etwa ein Fünftel aller Patienten mit Conjunctivitis gonorrhoica haben keine Genitalgonorrhöe.

5. Bezogen auf die Zahl der Fälle von Genitalgonorrhöe in den gleichen Altersstufen ist die Conjunctivitis gonorrhoica infantum etwa $45 \mathrm{mal}$ häufiger als die Conjunctivitis gonorrhoica der Erwachsenen.

6. Zwischen dem I5. und 32. Altersjahre findet sich eine allmähliche Abnahme der Conjunctivalgonorrhöe, bezogen auf die Genitalgonorrhöe.

7. Zwischen 32 and 50 Jahren wurden von uns keine Fälle von Conjunctivalgonorrhöe beobachtet.

8. Bezogen auf die Zahl der Fälle von Genitalgonorrhöe in den gleichen Alterssufen, ist die Conjunctivitis gonorrhoica jenseits des 50 . Altersjahres etwa $\mathrm{r}_{4}$ mal häufiger als zwischen

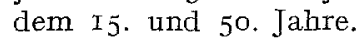

9. Aus diesen Zahlen ergibt sich, daß die Empfänglichkeit der Bindehaut für Gonokokkeninfektionen vom Lebensalter abhängig ist. Die Empfänglichkeit ist in der.Jugend vorhanden und nimmt dann allmählich ab, um nach dem 50. Altersjahre wieder zuzunehmen. Aus dieser Altersdisposition erklärt sich die Seltenheit der Conjunctivitis gonorrhoica im ganzen (geringe Emptänglichkeit zur Zeit des Maximums der Genitalgonorrhöe) und wenigstens teitweise die relative Häufigkeit von Conjunctivalgonorrhöe ohne Genitalgonorrhöe.

Literatur: ELSCHNIG, Gonorrhoische Erkrankungen des Auges. Handbuch der Geschlechtskrankheiten 2 (IOI2). - FEHR, Die gonorrhoischen Erkrankungen des Auges, Handbuch der Hautund Geschlechtskrankheiten 20, 2 (1930), - GROENoUw, Beziehungen der Allgemeinleiden und Organerkrankungen zu Veränderungen und Krankheiten des Sehorgans. Handbuch Graefe-Saemisch, 3. Aufl. I920. - HAUSTE1N, Statistik der Geschlechtskrankheiten. Handbuch der Haut- und Geschlechtskrankheiten 22 (1927). JAEGER, Die Geschlechtskrankheiten in der Schweiz. Ergebnisse der von der Schweiz. Vereinigung zur Bekämpfung der Geschlechtskrankheiten durchgeführten Enquête (I. Oktober I920 bis 30. September I92I). Bern: Büchler \& Co. 1923. - Косн u. СонN, Gonokokkeninfektionen. Handbuch der pathogenen Mikroorganismen. 4,2 (1928)

\section{ZUR FRAGE DER ENTSTEHUNG DER REINEN PIGMENTGALLENSTEINE.}

Von

Dr. Hermann Eitel.

Aus der Chirurgischen Universitätsklinik Freiburg i. BT. (Direktor: Professor Dr. E. REHN).

Durch Arbeiten von SchönheImer ${ }^{1}$, HAERKEL $^{1}$ und PEEL ${ }^{2}$ aus dem Aschoffischen Institut und HammarsteN ${ }^{3}$, HoppeSEYLER ${ }^{4}$ u. a. m. wurde bekannt, daß Schwermetalle (Kupfer, Zink, Mangan, Eisen) in erheblichem Maße in Gallensteinen angereichert sein können. Man fand bei Fällen von Hämochromatose, bei Fettieber und auch manchmal bei malignen Tumoren größere Kupfermengen in gleichzeitig vorhandenen Gallensteinen. Die Beobachtungen derartiger ,,Kupfersteine“ in vivo ist nicht sehr häufig. Die dazu führenden Erkrankungen bedürfen in der Regel der internen Behandlung, so da $\beta$ erst eine zufällig interkurrente Infektion der Gallenwege und ein dadurch bedingter chirurgischer Eingriff derartige Beobachtungen ermöglicht.

Deshalb sei in Folgendem ein solcher Zufallsbefund beschrieben, der vielleicht geeignet ist, auf Grund der anamnestischen Angaben einige Hinweise zur Entstehungsart dieser seltenen Steinformen $z u$ geben.

Eine ${ }_{5}^{6}$ jähr. Frau wird vom Arzt wegen anhaltender Schmerzen im rechten Oberbauch eingewiesen. Sie gibt in ihrer Vorgeschichte an, daß diese Beschwerden mit Unterbrechungen schon seit $3^{\circ} \mathrm{Jah}-$ ren beständen. Keine Gelbsucht. Die Zuckerbelastung ergab eine geringe Verzögerung des Blutzuckerabfalls, die Leber war stark vergrößert, die mit Tetragnost gefüllte Gallenblase klein; sie zeigte zahlreiche kleinere Steine. Diagnose: Chronische Cholecystitis mit Steinen.

Bei der Operation fand sich die Leber handbreit unter dem Rippenbogen. Sie hatte eine normal rote Farbe, keinerlei Zeichen von Fettleber und keine auffallende Pigmentierung. Ebenso wies der Darm keinerlei Pigmentierung auf, wie sie bei Hämochromatose mitunter zur Beobachtung kommt. Die Gallenblase war klein, geschrumpft und steinhaltig; keine akuten Entzündungserscheinungen.

Der pathologisch-anatomische Befund (Geh. Rat Aschoff) des eingesandten Präparates ergab u. a. folgendes: „Die Gallenblase enthält mehrere ganz schwarze, Stechapfelform zeigende, ziemlich derbe Konkremente. Sie müssen schon nach dem makroskopischen Aussehen als sog. „Kupfersteine“ betrachtet werden.

Es ist für die Frage der Entstehung dieser Steine folgendes von Interesse:

Die Patientin arbeitet seit ihrer frühesten Jugend im Rebbau, und zwar in einer Gegend, wo sehr viel Kupfervitriol gespritzt wird. Sie trinkt außerdem nach ihren Angaben im Tage etwa $1 / 21$,Haustrunlx", der bekanntermaßen einen erhöhten Kupfergehalt besitzt, was wir auch in diesem Falle durch Analysen bestätigen konnten.

Die Entstehungsursache der reinen Pigmentsteine (ihres relativ hohen Kupfergehaltes [0,3\%] wegen auch ,Kupfersteine" genannt) ist unbekannt. Man kennt lediglich die Krankheitsformen, bei denen sie gefunden zu werden pflegen. Diese sind eingangs erwähnt. Im vorliegenden Falle besteht nun weder eine Hämochromatose, noch Krebs in der Lebergegend, noch eine Fettleber, also keine der bisher in Verbindung mit Pigmentsteinen beobachteten pathologischen Veränderungen. Es ist hier die Möglichkeit nicht von đer Hand zu weisen, daß die jahrzehntelang anhaltende erhöhte Zufuhr von Kupfer ein begünstigender Faktor bei der Bildung dieser reinen Pigmentsteine gewesen ist.

Literatur: ${ }^{1}$ Klin. Wschr. I931, Nr 8, 345. - - 2 Hoppe-Seylers Z. I67, 250 (1927). - ${ }^{3}$ Iehrbuch der physiologischen Chemie. 4 Handbuch der physiologischen und pathologisch-chemischen Analyse. 5. Aufl. Berlin I883. S. 475

\section{UNTERSUCHUNGEN ÜBER DIE B. ZONDEKSCHE TRÄCHTIGKEITSREAKTION AUS DEM HARN BEI STUTEN.}

Von

Dr. W. SCHÄPER, Klein-Ziethen.

Aus dem Tierzuchtinstitut des Lehr- und Forschungsgutes der Tierärztlichen Hochschule Berlin in Klein-Ziethen (Direktor: Prof. Dr. STANG).

In dieser Wschr. I930, $\mathrm{Nr}_{\mathrm{r}} 49,2285-89$ berichtet BERNHARD ZONDEK u.a. über eine neue Trächtigkeitsdiagnose auf hormonalem Wege aus dem Harn von Stuten. Die von B. ZONDEK beschriebene diagnostische Reaktion beruht auf dem Nachweis der erhöhten Ausscheidung von zwei Hormonen* im Harn trächtiger Stuten, und zwar:

* Die Zondeksche hormonale Graviditatsreaktion beim Pferd beruht auf einem anderen Prinzip als die hormonale Schwangersclaftsrealtion beim Menschen (ASCHHEIMZONDEK). Die Schwangerschaftsreaktion bei der Frau berubt auf dem Nachweis von Hypophysenvorderlappenhormonen, wobei aber nur die HVR II und III, nicht die HVR I verwertet werden darf. Im Gegensatz dazu beruht die Zondeksche Graviditätsreaktion beim Pferd gerade auf dem Nachweis von HVR $I$ und vor allem auf dem Nachweis von Follikulin. Die bormonalen Reaktionen, die für die Gravi. ditätsdiagnose bei der Frau nicht verwertet werden dürfen, sind gerade für die Graviditätsdiagnose beim Pferd ausschlaggebend. 